\title{
The Link between Humidity and COVID-19 Caused Death
}

\author{
Katherine Li \\ Garnet Valley High School, Glen Mills, PA, USA \\ Email: katherineli@garnetvalley.org
}

How to cite this paper: Li, K. (2020) The Link between Humidity and COVID-19 Caused Death. Journal of Biosciences and Medicines, 8, 50-55.

https://doi.org/10.4236/jbm.2020.86005

Received: April 21, 2020

Accepted: May 23, 2020

Published: May 26, 2020

Copyright (C) 2020 by author(s) and Scientific Research Publishing Inc. This work is licensed under the Creative Commons Attribution International License (CC BY 4.0).

http://creativecommons.org/licenses/by/4.0/

\begin{abstract}
Objective: The objectives were to understand the relationship between humidity and COVID-19 fatality and to provide clues for improving COVID-19 patient survival rate. Methods: Humidity data and confirmed COVID-19 death cases for all fifty US states and Washington DC were collected and analyzed, and the relationship between humidity and COVID-19 mortality was derived. Results: There is a positive correlation between humidity and COVID-19 patient fatality. Conclusion: COVID-19 mortality directly and positively correlates with the humidity of the environment the COVID-19 virus experiences. Such correlation could be used to reduce COVID-19 patient fatality and save lives.
\end{abstract}

\section{Keywords}

COVID-19, Mortality, Humidity

\section{Introduction}

Since the novel coronavirus disease (COVID-19) broke out in Wuhan, China in December 2019, it has spread worldwide. The World Health Organization (WHO) reported that there are globally 693,282 confirmed cases and 33,106 death cases by March 30, 2020, resulting in a much higher death rate of 4.8\% from COVID-19 compared to the $0.1 \%$ typical death rate caused by the seasonal flu [1]. Driven by saving lives from the deadly COVID-19 pandemic, I wanted to investigate whether the death rate caused by COVID-19 could be reduced by controlling extrinsic environmental factors.

Weather conditions such as temperature and humidity can affect the transmission and virulence of viruses by impacting virus growth and host defense systems, and these effects have been studied extensively [2] [3] [4] [5]. However, those studies were mainly focused on virus transmission and little has been done 
on the effect of humidity on the death rate caused by viruses. Although high temperature has consistently been shown to decrease transmission of most viruses, humidity can either facilitate or inhibit viral transmission depending on the type of virus. I found three papers related to the effect of humidity on COVID-19 [6] [7] [8]. While two of the three papers only investigated the effect of humidity on COVID-19 transmission, Ma et al. did study the effect of humidity on death rate. However, Ma et al. attributed the effect of humidity on death rate to the impact on the patient defense system rather than on the environment humidity the virus experienced beforehand. In addition, Ma et al. only collected data from a single city (Wuhan, China) [8]. Ma's research inspired me to look at the impact of humidity on death rate in America. I was also curious to see whether the humidity the virus before infecting patients had any impact on death rate.

\section{Materials and Methods}

The number of total confirmed cases and death cases for COVID-19 were obtained from the New York Times website [9]. Typically, the most populous city or the state capitol was chosen for humidity data of each state. The average humidity data for 50 states and Washington DC reported by Weather Underground were used in this study [10]. As this study aimed to find the relationship between death rate and the humidity the virus experienced before infection, humidity data from a month prior to the studied cases were used to account for the delay between onset of transmission and death. Since the death cases used in this study are from March, the humidity data from the previous month were used. In detail, three data sets were collected: 1) COVID-19 data on March 22 and humidity data from January 22 to February 21;2) COVID-19 data on March 26 and humidity from January 26 to February 25;3) COVID-19 data on March 30 and humidity data in all of February. Death rate was calculated by dividing the number of death cases by the number of confirmed COVID-19 cases for each state or Washington DC. The average humidity is calculated by Equation (1), where $h_{i}$ stands for humidity on the $i$ th day and $n$ represents the total number of days during the period. Death rate in March vs average humidity in previous month was plotted for all 51 locations.

The correlation coefficient $r$ between humidity and death rate was calculated using Microsoft Excel function Correl based on the following Equation (2), where $x$ stands for COVID-19 mortality and $y$ represents average for each location.

$$
\begin{gathered}
\text { Average humidity }=\left(\sum_{i=1}^{n} h_{i}\right) / n \\
r=\frac{n\left(\sum x y\right)-\left(\sum x\right)\left(\sum y\right)}{\sqrt{\left[n \sum x^{2}-\left(\sum x\right)^{2}\right]\left[n \sum y^{2}-\left(\sum y\right)^{2}\right]}}
\end{gathered}
$$




\section{Results}

The correlation between the average humidity of the fifty states and Washington DC in late January and February along with the death cases data at three different dates in March 2020 is analyzed and the results are shown in Table 1 and Figure 1. There is a direct correlation between humidity and death rate caused by COVID-19 for all three data sets. The correlation coefficient is as high as 0.9 for the top 5 states with the most numbers of deaths. Although the correlation deceases when more states are included in the calculation, the correlation coefficient still remains as high as 0.5 even when the data for all fifty US states and Washington DC, including areas with zero reported death cases, were used (Table 1). This is not surprising, as when the number of deaths is small, the death rate is more prone to fluctuation due to other factors such as human migration. For example, when the stay-at-home order is placed and few people travel, the correlation coefficient for all fifty US states and Washington DC goes from 0.474 on March 22 to 0.516 on March 30. It is noticeable that the two driest states (Nevada and Arizona) and the most humid state (Alaska) are three consistent outliers (Figure 1). This observed digression from the correlation for these three states suggests a general correlation between humidity and death rate may not apply to extreme humidity values.

\section{Discussion}

The objective of this study is to illustrate the relationship between humidity and COVID-19 resultant death rate and thus reduce COVID-19 mortality by controlling humidity. The most recent report on humidity and COVID-19 mortality [8] was centered on the impact of humidity on the patients' defense systems. This study is focused on the effect of humidity on the COVID-19 virus itself and is accounted for by the time difference between humidity and COVID-19 death data, about one month apart. A significantly positive correlation between humidity and COVID-19 resultant death rate was observed. The mechanism behind this observed correlation between humidity and COVID-19 caused death rate is not clear. Comparative characterization of COVID-19 (such as gene sequencing, virulence analysis, etc.) that experienced different levels of humidity can help shed light on the underlying mechanism.

Table 1. The correlation between COVID-19 mortality and average humidity in previous month in the United States.

\begin{tabular}{ccccccc}
\hline State & Death case rank & Top 5 & Top 10 & Top 25 & Top 40 & 50 States/Washington DC \\
\hline Mar. & Death cases & $27-157$ & $10-157$ & $1-157$ & $0-157$ & $0-157$ \\
22 & Correlation $(r)$ & 0.832 & 0.734 & 0.533 & 0.471 & 0.474 \\
Mar. & Death cases & $62-385$ & $19-385$ & $7-385$ & $1-385$ & $0-385$ \\
26 & Correlation $(r)$ & 0.874 & 0.772 & 0.546 & 0.494 & 0.421 \\
Mar. & Death cases & $186-1244$ & $56-1244$ & $18-1244$ & $4-1244$ & $0-1244$ \\
30 & Correlation $(r)$ & 0.906 & 0.840 & 0.649 & 0.495 & 0.516 \\
\hline
\end{tabular}

Note: Three outliers (AK, AZ and NV) were excluded from the correlation coefficient $(r)$ calculation. 
(a)

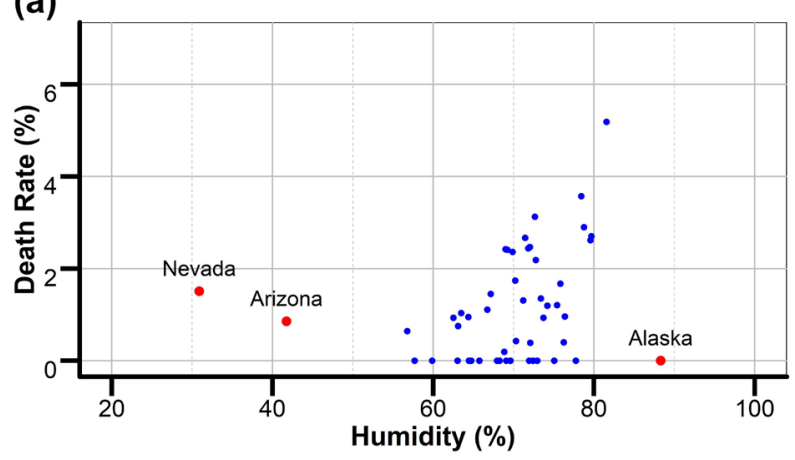

(b)

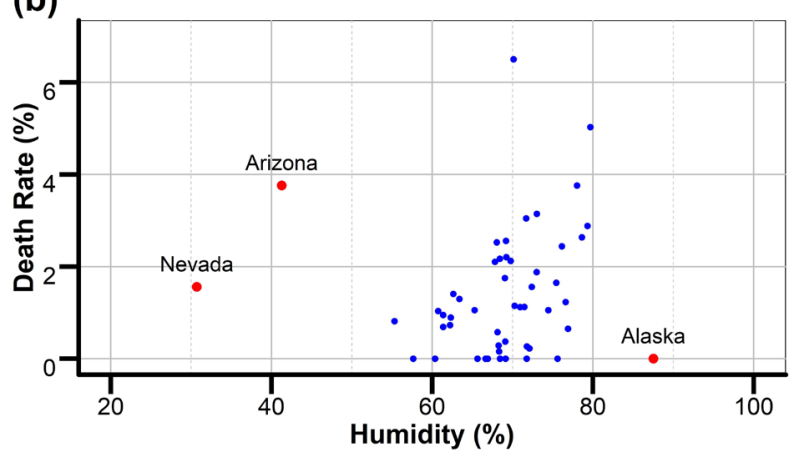

(c)

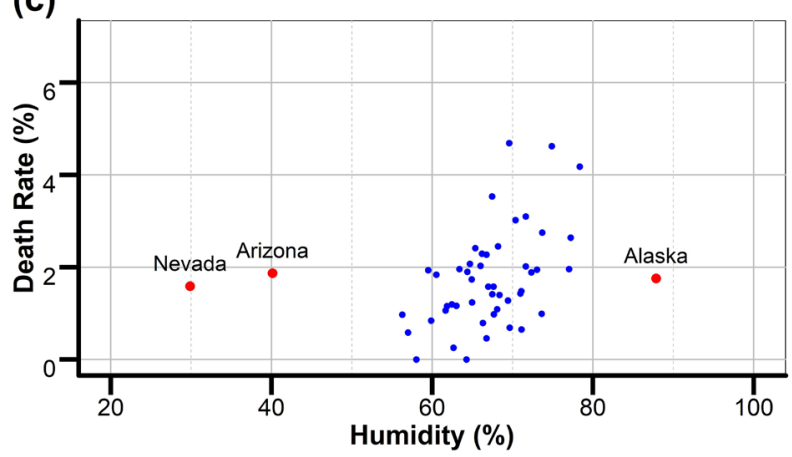

Figure 1. Mortality in March resulted from COVID-19 and local humidity in February. Each data point represents a data set from a state or Washington DC from America. Except three consistent outliers, there is a clear correlation between humidity and COVID-19 resultant death rate. (a) COVID-19 mortality on March 22 and average humidity from January 22 to February 21; (b) COVID-19 mortality on March 26 and average humidity from January 26 to February 25; (c) COVID-19 mortality on March 30 and average humidity in February.

Three outliers (the two driest states, Nevada and Arizona, and the most humid state, Alaska) have been observed consistently in all three data sets. One possible explanation is that the correlation between humidity and COVID-19 mortality may only be significant within a certain range of humidity as it has been found before that some viruses have a narrow ideal humidity range [4]. A more insightful explanation may rely on the mechanism study of the effect of humidity on COVID-19 virulence. 
I also found that the correlation increases with the rise in number of death cases. When the number of deaths is small, one or two deaths from a random event weighs heavily in the mortality calculation and thus twists the correlation. For example, a very sick COVID-19 patient may have moved from one state to another state, in which no COVID-19 deaths have been recorded yet, and later dies in the state he just moved to. The impact of human migration could explain why data collected at the end of March showed a better overall correlation than the data collected earlier since more people started to stay home towards the end of March.

This study was conducted at a large scale (statewide) but localized studies may be more informative on how to reduce COVID-19 fatality. State is an administrative unit and humidity within a state, particularly a large state, can vary a lot. Therefore, states may not be the best geographic unit to study the effect of humidity on COVID-19 fatality. Towns, communities, and even residential buildings may be better geographic units for a more comprehensive study in populated areas if localized data is available.

In the meantime, a couple of measures can be immediately taken that can potentially save lives: First, hospitals and residential houses can lower their humidity setting to a certain range. Second, vulnerable people should stay in areas of lower humidity.

The reason that I did not study the correlation between temperature and COVID-19 mortality is the obviousness that high temperature decreases transmission, livability, and viral fatality as reported by many scientists [3] [4] [5] [7] [8]. I did not find a strong correlation between temperature and COVID-19 fatality (data not shown). However, temperature and humidity may not affect the virus independently [3] [4] [5] [7] [8]. A comprehensive study of all environmental parameters (temperature, humidity, etc.) on COVID-19 mortality deserves further scrutiny. In addition, a more extensive collection of data, including a comprehensive analysis of other potential explanatory variables, like ages of patients, could be more informative.

\section{Conclusion}

This study is the first one to investigate the effect of humidity on the cumulative fatality of COVID-19 for all fifty US states and Washington DC using publicly accessible data. My analysis suggests that there is a direct and positive correlation between COVID-19 mortality and the humidity of the environments the virus experiences. Such findings could help with COVID-19 virulence and potentially reduce COVID-19 fatality.

\section{Acknowledgements}

My father, Yougen Li, for his discussion and input.

\section{Conflicts of Interest}

The author declares no conflicts of interest regarding the publication of this paper. 


\section{References}

[1] WHO (2020) Coronavirus Disease 2019 (COVID-19) Situation Report 70.

[2] Kudo, E., et al. (2019) Low Ambient Humidity Impairs Barrier Function and Innate Resistance against Influenza Infection. Proceedings of the National Academy of Sciences, 116, 10905-10910. https://doi.org/10.1073/pnas.1902840116

[3] Lowen, A. and Steel, J. (2014) Roles of Humidity and Temperature in Shaping Influenza Seasonality. Journal of virology, 88, 7692-7695. https://doi.org/10.1128/JVI.03544-13

[4] Price, R., Graham, C. and Ramalingam, S. (2019) Association between Viral Seasonality and Meteorological Factors. Scientific Reports, 9, Article No. 929. https://doi.org/10.1038/s41598-018-37481-y

[5] Memarzadeh, F. (2011) Literature Review of the Effect of Temperature and Humidity on Viruses. ASHRAE Transactions, 117, Part 2: ML-11-024.

[6] Luo, C., et al. (2020) Possible Transmission of Severe Acute Respiratory Syndrome Coronavirus 2 (SARS-CoV-2) in a Public Bath Center in Huai'an, Jiangsu Province, China. JAMA Network Open, 3, e204583.

https://doi.org/10.1001/jamanetworkopen.2020.4583

[7] Bukhari, Q. and Jameel, Y. (2020) Will Coronavirus Pandemic Diminish by Summer? https://doi.org/10.2139/ssrn.3556998

[8] Ma, Y., et al. (2020) Effects of Temperature Variation and Humidity on the Mortality of COVID-19 in Wuhan. https://doi.org/10.1101/2020.03.15.20036426

[9] https://www.nytimes.com/interactive/2020/us/coronavirus-us-cases.html

[10] https://www.wunderground.com/history 